\title{
Rapid, High-Level Transient Expression of Papillomavirus-Like Particles in Insect Cells
}

BioTechniques 20:890-895 (May 1996)

\author{
Diana Benincasa, Melvin \\ Silberklang, George E. Mark \\ III and Steven W. Ludmerer \\ Merck Research Laboratories, \\ Rahway, NJ, USA
}

\section{INTRODUCTION}

Many aspects of protein structural analysis depend upon the expression of a large number of properly modified or folded variant sequences to screen for function (see, for example, Reference 20). In some cases, sufficient quantities of full-length peptide can be recovered by in vitro transcription/translation, but proper folding cannot be controlled $(1,16)$. Short sequences, such as singlechain Fv antibodies, can be expressed and "displayed" in bacteria or in filamentous phage in partially or even completely folded form for screening purposes (23). However, larger proteins of mammalian origin often require expression in eukaryotic cells for proper folding and assembly. Furthermore, a high local concentration of expressed protein monomers can be important in proper folding, particularly in the case of assembly of viral coat proteins. Because transient transfection of mammalian cells such as Cos or 293 is relatively inefficient and low-yielding, screening large numbers of variant sequences by these means can prove quite laborious $(8,9)$. One system that overcomes some of these difficulties is the insect cell baculovirus gene expression vector system (19), which has, for example, proven very popular for generation of virus-like particles (VLPs) $(7,22)$. Nevertheless, a significant drawback of this system is the relatively long time required to obtain plaque-purified, expanded recombinant baculoviral stocks with which to express useful quantities of VLPs. Even the most time-efficient baculovirus system reported to date, the bacmid system, requires two weeks from initial transfection to recovery of screenable product (15).
We have an interest in identifying the amino acid residues that confer antigenic type-specificity on human papillomavirus (HPV) subtypes $(2,4)$. Many of the type-specific epitopes are conformationally dependent and are detectable only upon VLP assembly. The L1 structural coat protein of several animal and human papillomaviruses efficiently self-assembles when expressed in insect cells by means of recombinant baculovirus (13). The time and labor involved in the generation of pure recombinant virus precludes the use of this method to screen a large number of VLP variants produced through site-directed mutagenesis. However, we previously observed that, when expressed in the baculovirus system, recombinant proteins such as antistasin and trigrammin were detectable as secreted products in $\mu \mathrm{g} / \mathrm{mL}$ quantities within 5-7 days of the initial transfection of insect cells with plasmid and viral DNAs $(6,10)$. Based upon this observation, we examined whether sufficient quantities of papillomavirus L1 protein would accumulate to allow selfassembly into VLPs upon transient expression, particularly if a more efficient baculovirus transfection system, such as the BaculoGold ${ }^{\mathrm{TM}}$ system, were utilized. Employing a rapid 6-day transient transfection protocol, we report the production of L1 coat protein of cotton-tail rabbit papilloma virus (CRPV) and HPV11, both properly assembled into VLPs. Extracts prepared from transiently transfected cells contained immunogenic material recognized by type-specific and VLP-dependent monoclonal antibodies generated against either CRPV or HPV11 VLPs. The transiently expressed material was not cross-reactive with other type-specific antibodies, and recognition was 
sensitive to alkaline denaturation. The insect-cell-based transient expression system we describe should prove useful in many other high-throughput protein sequence screening applications where high-level expression, coupled with high-fidelity folding and assembly, are required.

\section{MATERIALS AND METHODS}

\section{Transient Expression of VLPs in Sf9 Cells}

The HPV11 L1 structural gene was cloned from clinical isolates using the polymerase chain reaction (PCR) with primers designed from the published L1 sequence $(5,12)$. The CRPV L1 structural gene was cloned by PCR from viral genomic DNA and was the generous gift of Drs. Kathrin Jansen and Loren Schultz (Merck Research Laboratories, West Point, PA, USA). The L1 genes were subcloned into pVL1393 (Stratagene, La Jolla, CA, USA) for expression in Sf9 cells.

Sf9 cells were cotransfected using the BaculoGold ${ }^{\mathrm{TM}}$ Transfection kit (Pharmingen, San Diego, CA, USA) according to the manufacturer's instructions with the following modification: $8 \times 10^{6} \mathrm{Sf} 9$ cells were transfected in a $100-\mathrm{mm}$ dish, with $4 \mu \mathrm{g}$ of BaculoGold viral DNA and $6 \mu \mathrm{g}$ of test plasmid DNA. Cells were harvested after 6 days, except where otherwise specified, and assayed for VLP production by Western blot or enzyme-linked immunosorbent assay (ELISA) assay (11).

\section{Preparation of Sf9 Extracts and ELISA Assays}

Six days after transfection, the plates were scraped to resuspend cells, and the cells were collected by lowspeed centrifugation. Cells were resuspended in $300 \mu \mathrm{L}$ of breaking buffer (1 $\mathrm{M} \mathrm{NaCl}, 0.02 \mathrm{M}$ Tris-HCl pH 7.6) and homogenized for $30 \mathrm{~s}$ on ice using a Polytron PT 1200 B with a PT-DA 1205/2-A probe (Brinkman Instruments, Westbury, NY, USA) in a Falcon 2059 tube. Samples were spun at 2500 rpm in a GPR centrifuge (Beckman Instruments, Fullerton, CA, USA) for 3 min to pellet debris. The tubes were washed with an additional $150 \mu \mathrm{L}$ of breaking buffer, and the supernatants were collected in 1.5-mL microcentrifuge tubes, and respun for $5 \mathrm{~min}$ in an Eppendorf ${ }^{\circledR}$ microcentrifuge (Model 5415; Brinkman Instruments). ELISA assays were begun the same day.

Five microliters of extract were diluted in $50 \mu \mathrm{L}$ of $1 \%$ bovine serum albumin (BSA) in phosphate-buffered saline (PBS) solution, aliquoted onto a 96-well Immulon ${ }^{\circledR} 2$ ("U" bottom) microtitration plate (Dynatech Laboratories, Bethesda, MD, USA) and incubated overnight at $4^{\circ} \mathrm{C}$. Extracts were removed and the plate was blocked with $5 \%$ powdered milk/PBS. All subsequent wash steps were performed with $1 \%$ BSA/PBS. The plate was incubated at room temperature with primary antibody for $1 \mathrm{~h}$. Primary monoclonal antibodies CRPV.5A, H6.C6 and H11.F1 were obtained as ascites stock from Dr. Neil Christensen (Pennsylvania State University). CRPV.5A and H11.F1 are VLP-dependent and type-specific antibodies that recognize CRPV and HPV11 VLPs, respectively $(3,4)$. Monoclonal antibody H6.C6, raised against HPV6 VLPs, recognizes a linear outer epitope that is present in both denatured L1 samples and VLPs. Furthermore, the antibody is cross-reactive with HPV11 (N. Christensen, personal communication). They were diluted $10^{5}$-fold in $1 \%$ BSA/PBS before use. After washing in 1\% BSA/ $\mathrm{PBS}$, the plates were incubated for $1 \mathrm{~h}$ with secondary antibody, horse radish peroxidase-labeled Goat anti-Mouse IgG $(\gamma)$ (Kirkegaard \& Perry Laboratories, Gaithersburg, MD, USA), diluted $10^{-3}$ in $1 \%$ BSA in PBS. After a final washing, the 2,2'-azino-di(3-ethylbenzthiazoline) sulfonic acid substrate (Zymed Laboratories, South San Francisco, CA, USA) was added and absorbance read at $405 \mathrm{~nm}$.

\section{RESULTS}

\section{Expression of CRPV L1 is Maximal After Six Days}

To determine the time required for maximal expression, plasmid p1393: CRPV was co-transfected with BaculoGold DNA into SF9 cells and plates were incubated at $28^{\circ} \mathrm{C}$ for $3,4,5,6$ and 7 days. Plates were scraped, cells collected by low-speed centrifugation, resuspended in $200 \mu \mathrm{L}$ of Laemmli loading buffer and heated for $5 \mathrm{~min}$ at $95^{\circ} \mathrm{C}$. Twenty-microliter fractions were electrophoretically separated under denaturing conditions, transferred to nitrocellulose and probed with a commercially available rabbit polyclonal antiserum to CRPV L1 (Dako Corporation, Carpenteria, CA, USA).

Although a number of cross-reactive bands are visible (Figure 1), there is a band, which appears only in the pVL1393:CRPV fractions, that runs at the expected molecular weight of 55 $\mathrm{kDa}$, which is consistent with the intact CRPV L1 protein. The intensity of this band increases to a maximum in the six-day sample. The other detectable bands are also observed with a wildtype baculovirus control extract and undoubtedly represent protein species cross-reactive with the polyclonal serum. We conclude that, in this system, L1 production peaks after six days. A denaturing SDS-PAGE Western blot assay for L1 was preferred over an ELISA assay because it can be used to specifically measure the intact L1 polypeptide chain, which represents the primary self-assembling species. By comparison to a standard curve of purified L1, we estimate L1 production to be $1-2 \mu \mathrm{g} \mathrm{L1}$ per $100-\mathrm{mm}$ plate after six days (data not shown).

\section{Transiently Expressed L1 Assembles into VLPs That are Detected by Type-Specific Antibodies}

The virus particle is comprised of 72 pentameric capsomers of L1. Several reports have demonstrated that baculovirus expression of L1 from diverse papillomavirus types supports not only L1 production, but also assembly into a higher order structure called a viruslike particle (VLP), which is indistinguishable from native virus in electronmicrograph studies (13). Furthermore, many type-specific epitopes are conditional upon VLP formation for proper conformation and display $(3,4)$. Therefore, it was important to demonstrate that transient expression in $\mathrm{Sf9}$ cells supports VLP assembly.

We prepared extracts from Sf9 cells 
transiently transfected with either pVL1393:CRPV or pVL1393:11 and evaluated their ability to elicit binding by anti-L1 monoclonal antibodies dependent upon VLP conformation and specific for either CRPV or HPV11 VLPs (Figure 2). Figure 2A shows the results of the ELISA with the CRPV-specific monoclonal antibody CRPV.5A. A strong signal is produced with either purified CRPV VLPs or pVL1393:CRPV extracts. No binding above background level is observed with either purified HPV11 VLPs or pVL1393:HPV11 extracts from transfection.

The results are reversed when the ELISA is performed with HPV11-specific monoclonal antibody H11.F1 (Figure 2B). A strong signal is ob- served with either purified HPV11 VLPs or extract from transfection of pVL1393:HPV11. The signal produced with either pure CRPV VLPs or pVL1393:CRPV extracts is not above background.

To confirm that binding was VLP dependent, we evaluated binding of monoclonal antibody H11.F1 to a denatured extract (Figure 3). An aliquot of HPV11 SF9 extract was diluted into 0.1 $\mathrm{M}$ sodium carbonate, $\mathrm{pH} 10.5$, and incubated at room temperature for $1 \mathrm{~h}$. The extract was coated onto a microplate and allowed to dry. In parallel, untreated pVL1393:HPV11 extract was coated onto a microplate and incubated overnight at $4^{\circ} \mathrm{C}$. The presence of $\mathrm{L} 1$ was verified with antibody H6.C6. Figure 3 shows that the strong signal elicit-

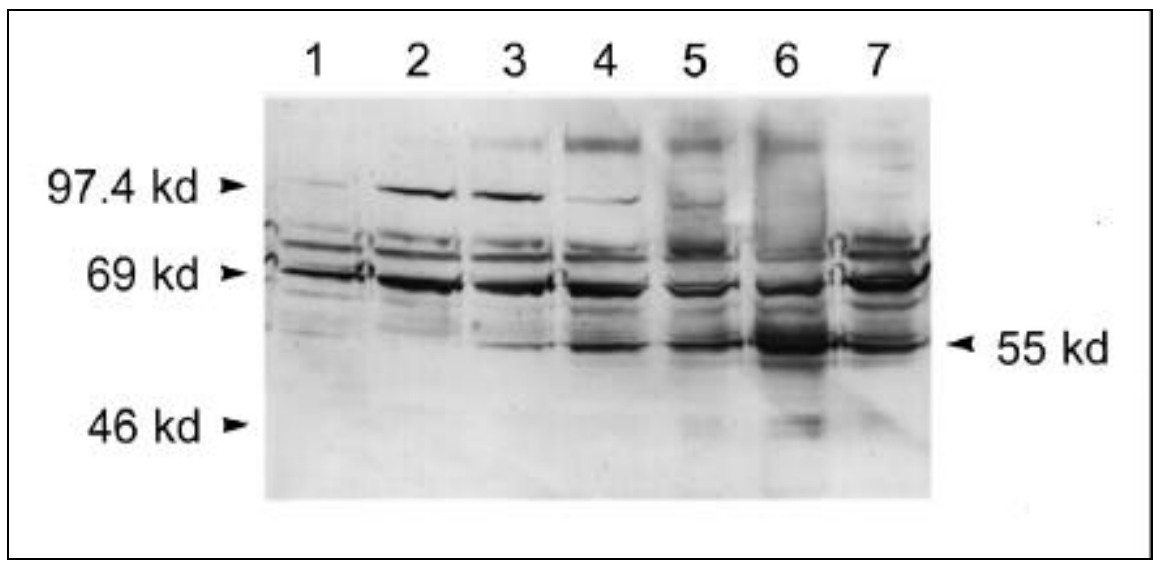

Figure 1. Production of CRPV L1 is maximal 6 days posttransfection. Sf9 cells were cotransfected with pVL1393:CRPV and BaculoGold DNA. Samples were harvested at various time points and analyzed by immunoblot with a polyclonal antibody against CRPV L1 as described in the text. Lane 1, baculovirus DNA, 7 days; lane 2, no DNA, 7 days; lane 3, pVL1393:CRPV, 3 days; lane 4, pVL1393:CRPV, 4 days; lane 5, pVL1393:CRPV, 5 days; lane 6, pVL1393:CRPV, 6 days; and lane 7, pVL1393:CRPV, 7 days.
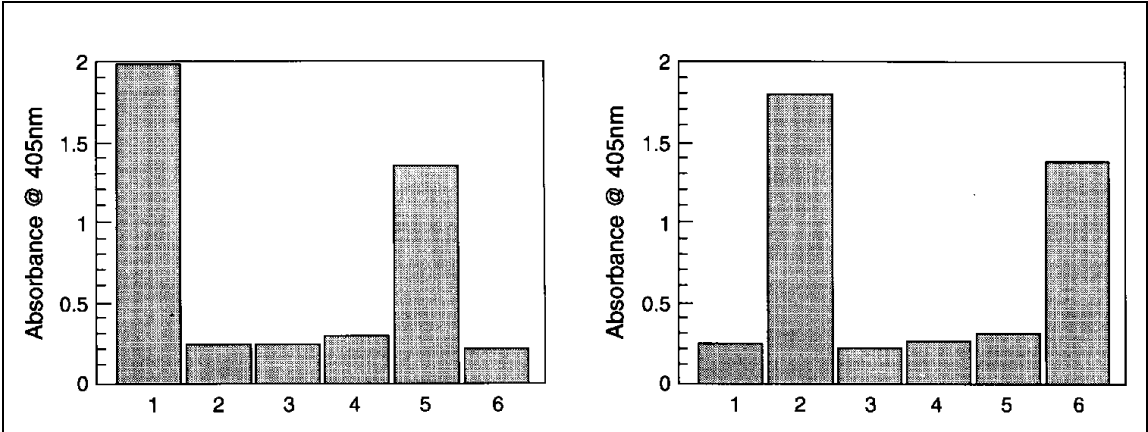

Figure 2. VLPs with type-specific properties are generated in transient transfection. Sf9 cells were cotransfected with BaculoGold DNA and either pVL1393:CRPV or pVL1393:HPV11. Cells were harvested after six days, extracts prepared and ELISAs performed as described in the text. Column $1,1 \mu \mathrm{g}$ CRPV VLPs; column 2, $1 \mu \mathrm{g}$ HPV11 VLPs; column 3, SF9 extract; column 4, baculovirus DNA; column 5, pVL1393:CRPV; column 6, pVL1393:HPV11. A. Primary antibody is $10^{-5}$ dilution of CRPV.5A ascites fluid. B. Primary antibody is $10^{-5}$ dilution of H11.F1 ascites fluid. ed by pVL1393:HPV11 extracts is reduced to background level in the alkaline-treated sample. In contrast, alkaline treatment had no effect on recognition by control monoclonal H6.C6.

The results demonstrate that transient expression in Sf9 cells supports production of L1 species that contain type-specific epitopes known to be dependent upon VLP formation for display. We have also successfully expressed HPV6 L1 VLPs in this system (data not shown).

\section{DISCUSSION}

In this work, we have confirmed that the level of papillomavirus L1 protein expression obtained in Sf9 cells cotransfected with a pVL1393-based vector and BaculoGold viral DNA is sufficient within 6 days posttransfection to generate analytical levels of self-assembled VLPs. Expression of L1 protein appears to reach about $1-2 \mu \mathrm{g} / 100-$ $\mathrm{mm}$ petri dish, easily enough VLP material from a single transfection for epitope expression screening using an ELISA format. We have applied this technique to screen sequence variants produced by site-directed mutagenesis and have succeeded in identifying

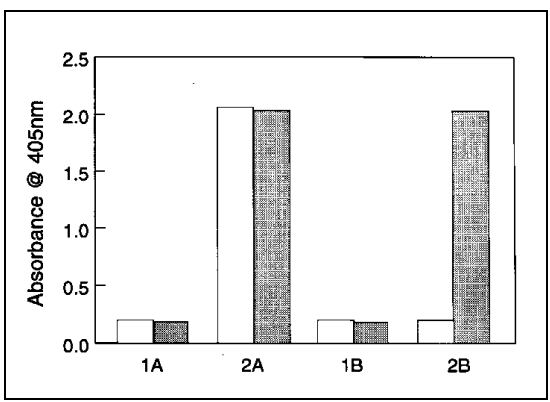

Figure 3. Immunogenic material produced by transient transfection is sensitive to denaturation. Sf9 cells were cotransfected with pVL1393: HPV11 and BaculoGold DNA, cells were harvested after six days and extracts prepared as described in the text. A portion of the extracts were denatured by dilution into $0.1 \mathrm{M}$ sodium carbonate, $\mathrm{pH} 10.5$, and incubated at room temperature for $1 \mathrm{~h}$. These extracts were then coated onto a microplate and allowed to dry. Untreated extracts were coated onto microplates and incubated overnight at $4^{\circ} \mathrm{C}$. ELISAs were performed as described in Materials and Methods using a $10^{-5}$ dilution of either H11.F1 ( $\square$ ) or H6.C6 (ם) ascites. Column 1, Sf9 extract; column 2, pVL1393: HPV11 extract. A, extract is nondenatured. B, extract was carbonate buffer treated. 


\section{Research Reports}

critical residues that comprise a conformationally dependent epitope in human papillomavirus 11 (2).

It had previously been noted that detectable levels of recombinant protein appear within a few days of baculovi$\mathrm{ral} /$ plasmid DNA cotransfection $(6,10$, $18,24,25)$. This "transient" expression was used principally to confirm expression of the desired protein. In other work, "transient" expression has been exploited to study baculovirus transcription by linking the promoter to the bacterial reporter gene chloramphenicol transacetylase (CAT) on plasmidborne vectors $(17,21,24,25)$. However, other applications of the baculovirus expression system, such as the study of viral structural protein self-assembly or various aspects of protein structure/ function analysis and engineering, have continued, for the most part, to use infection with plaque-purified virus to produce the desired protein(s).
It is in this context that we have found that the BaculoGold system generates transient expression levels of a viral structural protein high enough to satisfy even the stringent test of selfassembly, a concentration-dependent process. These levels of expressed protein are achieved within 6 days posttransfection, considerably faster than reported for the next quickest baculovirus recombinant viral isolation system, the bacmid system (15). The expression levels are also comparable to the best levels obtainable in mammalian cell transient expression systems. This system is well-suited for inquiries that require only analytical amounts of material that must be properly folded or assembled (2). We believe that it holds promise for investigations into structural parameters on nonviral proteins as well.

The intracellular events that follow DNA cotransfection, recombination be- tween viral and plasmid DNA and expression of plasmid-borne genes, are not well understood. Our results and other published reports that document the gradual kinetics of recombinant protein expression between days 3 and 6 posttransfection are consistent with a hypothesis that both transient expression from tranfected plasmid DNA and expression from recombinant viralborne genes in newly infected cells contribute to the total protein yield. We observed optimal levels 5-6 days posttransfection, when essentially all the cells show signs of infection, and when the concentration of infectious viral particles in the supernatant is over $10^{6} / \mathrm{mL}$ (A. Lenny and M. Silberklang, unpublished observations). Because almost all infectious virus produced by the BaculoGold system is recombinant (14), much of the late gene expression (driven by the polyhedrin promoter) observed may be due to infection of 
cells by newly released recombinant virus. In this sense, the observed expression is not much different from what would occur with plaque-purified virus. Therefore, the transient system we describe provides a very practical avenue to screen sequence variants with the baculovirus system, and should be applicable to all cases where microgram quantities of expressed protein are sufficient to evaluate results. It additionally offers a key advantage over other transient systems in that further scale-up for production of any particular protein sequence of interest would simply involve expansion by standard methods, from the viral progeny of the transient cotransfection, of a pure recombinant baculoviral stock.

\section{REFERENCES}

1.Anderson, C.W., J.W. Straus and B.S. Dudock. 1983. Preparation of a cell-free proteinsynthesizing system from wheat germ. Meth. Enzymol. 101:635-650.

2.Benincasa, D., G.F. Hollis, J. Hollis, G.E. Mark III and S.W. Ludmerer. 1995. Epitope mapping of the neutralizing epitope of HPV11. 14th International Papillomavirus Conference. Quebec City, Canada. p. 205.

3.Christensen, N.D. and J.W. Kreider. 1991. Neutralization of CRPV infectivity by monoclonal antibodies that identify conformational epitopes on intact virions. Virus Res. 21:169179.

4.Christensen, N.D., J.W. Kreider, N.M. Cladel, S.D. Patrick and P.A. Welsh. 1990. Monoclonal antibody-mediated neutralization of infectious human papillomavirus type 11. J. Virol. 64:1936-1944.

5.Dartmann, K., E. Schwarz, L. Gissmann and H. zur Hausen. 1986. The nucleotide sequence and genome organization of human papilloma virus Type 11. Virology 151:124130.

6.Daugherty, B.L., S.M. Zavodny, A.B. Lenny, M.A. Jacobsen, R.W. Ellis, S.W. Law and G.E. Mark. 1990. The uses of computer-aided signal peptide selection and polymerase chain reaction in gene construction and expression of secreted proteins. DNA Cell Biol. 9:453-459.

7.French, T.J. and P. Roy. 1990. Synthesis of bluetongue virus (BTV) corelike particles by a recombinant baculovirus expressing the two major structural core proteins of BTV. J. Virol. 64:1530-1536.

8.Gluzman, Y. 1981. SV40-transformed simian cells support the replication of early SV40 mutants. Cell 23:175-182.

9.Graham, F.L., P.S. Abrahams, C. Mulder, H.L. Heijneker, S.O. Warnaar, F.A. deVries, W. Fiers and A.J. van der Eb. 1974. Studies on the in vitro transformation by DNA and DNA fragments of human adenoviruses and simian virus 40. Cold Spring Harbor
Symp. Quant. Biol. 39:637-650.

10.Han, J.H., S.W. Law, P.M. Keller, P.J. Kniskern, M. Silberklang, J.-S. Tung, T.B. Gasic, G.J. Gasic, P.A. Friedman and R.W. Ellis. 1989. Cloning and expression of cDNA encoding antistasin, a leech-derived protein having anti-coagulant and anti-metastatic properties. Gene 75:47-57.

11.Harlow, E. and D. Lane. 1988. Antibodies: A Laboratory Manual. Cold Spring Harbor Press, Cold Spring Harbor, NY.

12.Innis, M.A., D.H. Gelfand, J.J. Sninsky and T.J. White. 1990. PCR Protocols. A Guide to Methods and Applications. Academic Press, San Diego, CA.

13.Kirnbauer, R., F. Booy, N. Cheng, D.R. Lowy and J.T. Schiller. 1992. Papillomavirus L1 major capsid protein self-assembles into virus-like particles that are highly immunogenic. Proc. Natl. Acad. Sci. USA 89:1218012184.

14.Kitts, P.A. and R.D. Possee. 1993. A method for producing recombinant baculovirus expression vectors at high frequency. BioTechniques 14:810-817.

15.Luckow, V.A., S.C. Lee, G.C. Barry and P.O. Olins. 1993. Efficient generation of infetious recombinant baculoviruses by site specific transposon-mediated insertion of foreign genes into a baculovirus genome propagated in Escherichia coli. J. Virol. 67:4556-4579.

16.Melton, D.A., P.A. Krieg, M.R. Rebagliate, T. Maniatis, K. Zinn and M.R. Green. 1984 Efficient in vitro synthesis of biologically active RNA and RNA hybridization probes from plasmids containing a bacteriophage SP6 promoter. Nucleic Acids Res. 12:7035-7056.

17.Nissen, M.S. and P.D. Friesen. 1989. Molecular analysis of the transcriptional regulatory region of an early baculovirus gene. J. Virol. 63:493-503.

18.Ooi, B.G., C. Rankin and L.K. Miller. 1989. Downstream sequences augment transcription from the essential initiation site of a baculovirus polyhedrin gene. J. Mol. Biol. 210:721-736

19.O'Reilly, D.R., L.K. Miller and V.A. Luckow. 1992. Baculovirus Expression Vectors: A Laboratory Manual. W.H. Freeman and Company, New York.

20.Pabo, C.O. and R.T. Sauer. 1992. Transcription factors: structural families and principles of DNA recognition. Annu. Rev. Biochem. 61:1053-1085.

21.Rankin, C., B.G. Ooi and L.K. Miller. 1988 Eight base pairs encompassing the transcriptional start point are the major determinant for baculovirus polyhedrin gene expression. Gene 70:39-49.

22.Takehara, K., D. Ireland and D.H.L. Bishop. 1988. Co-expression of the hepatitis B surface and core antigens using baculovirus multiple expression vectors. J. Gen. Virol. 69:2763-2777.

23.Ward, E.S. 1992. Expression and purification of antibody fragments using Escherichia coli as a host, p. 121-137. In C.A.K. Borrebaeck (Ed.), Antibody Engineering, a Practical Guide. W.H. Freeman and Company, New York

24.Weyer, U. and D. Possee. 1988. Functional analysis of the P10 gene $5^{\prime}$ leader sequence of the Autographa californica nuclear polyhedrosis virus. Nucleic Acids Res. 16:36353653

25.Weyer, U. and D. Possee. 1989. Analysis of the promoter of the Autographa californica nuclear polyhedrosis virus p10 gene. J. Gen. Virol. 70:203-208.

Received 24 August 1995; accepted 2 November 1995

Address correspondence to:

Steven W. Ludmerer

Merck Research Laboratories

P.O. Box 2000

Rahway, NJ 07065, USA 